\title{
Design of Plasmon Resonance Shifts by the Galvanic Replacement Degree of Au-Ag
}

\section{Nanozappers}

\author{
Hung-Chi Yen, ${ }^{\neq 1}$ Man-Nung Su, ${ }^{\neq 2}$ You-Cheng Liu, ${ }^{1}$ Ming-Wei \\ Lee, ${ }^{1,2}$ Yae-Lin Sheu, ${ }^{2}$ Liang-Yan Hsu, ",2 and Chia-Chun \\ Chen ${ }^{*}, 1,2$
}

${ }^{1}$ Department of Chemistry, National Taiwan Normal University, Taipei 116, Taiwan, ${ }^{2}$ Institute of Atomic and Molecular Sciences, Academia Sinica, Taipei 106, Taiwan

\section{Estimation of the added $\mathrm{HAuCl}_{4}$ equivalent ratio to $\mathrm{Ag}$}

Because the absorbance of the Au nanoparticles solution at $400 \mathrm{~nm}$ is mainly contributed from the interband transitions in the metallic $\mathrm{Au},{ }^{1}$ the $\left[\mathrm{Au}^{0}\right]$ concentration can be directly determined from the calibration line (Figure S1), ${ }^{2}$ regardless of the shape and size of the Au nanoparticles. To estimate the $\left[\mathrm{Au}^{0}\right]$ concentration from the as-prepared Au nanorods solution, the absorbance value at the wavelength of $400 \mathrm{~nm}$ was recorded with a UV-Vis spectrometer, and the $\left[\mathrm{Au}^{0}\right]$ concentration corresponding to each $\mathrm{Au}$ nanorods sample was measured by inductively coupled plasma mass spectrometry (ICP-MS).

The Ag/Au molar ratio of Au@Ag nanocuboids was estimated from the following

equation, $\quad \frac{M_{A g}}{M_{A u}}=\frac{\frac{\left(V_{A g}-V_{A u}\right) \times d_{A g}}{m_{A g}}}{\frac{V_{A u} \times d_{A u}}{m_{A u}}}$

(Equation S1)

where $M_{A g}$ was the mole of $A g, M_{A u}$ was the mole of $A u,\left(V_{A g}-V_{A u}\right)$ implied the average volume of Au@Ag nanocuboids subtracted the space occupied by the central $\mathrm{Au}$ nanorod, $\mathrm{d}_{A g}$ was the density of $A g, \mathrm{~d}_{A u}$ was the density of $A u, \mathrm{~m}_{A g}$ and $\mathrm{m}_{A u}$ was the atomic mass of $A g$ and $A u$, respectively. The average volume of $A u$ nanorods and Au@Ag nanocuboids was evaluated form the corresponding TEM images. 


\section{Effective dielectric functions for the filling materials in Au-Ag nanozappers}

The effective dielectric function $\left(\epsilon_{\mathrm{eff}, x}\right)$ is expressed by using the refractive index of effective material,

$\epsilon_{\mathrm{eff}, x}=\left(n_{\mathrm{eff}, x}+i k_{\mathrm{eff} . x}\right)^{2}$

, where $n_{\text {eff, } x}$ and $k_{\text {eff. } x}$ denote the real part and imaginary part of refractive index, respectively. The effective refractive index is the linear combination of those of $\mathrm{Ag}$ and water,

$n_{\mathrm{eff}, x}=n_{\mathrm{Ag}}(1-x)+1.33 x$

(Equation S3)

$k_{\mathrm{eff}, x}=k_{\mathrm{Ag}}(1-x)$

(Equation S4)

, where $x$ denotes the ratio of water in the effective material. $n_{\mathrm{Ag}}$ and $k_{\mathrm{Ag}}$ in the equations is directly from the experimental data of $\mathrm{Ag}$ we mentioned above.

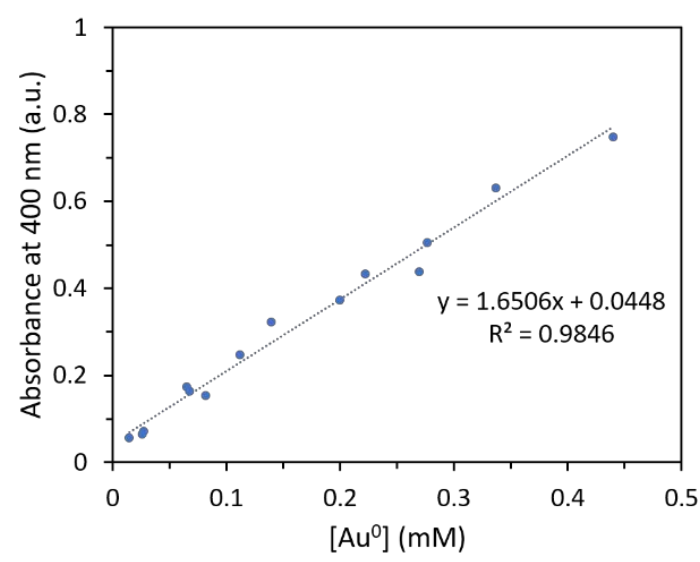

Figure S1. Calibration curve of $\left[\mathrm{Au}^{0}\right]$ concentration plotted as a function of the absorbance of $\mathrm{Au}$ nanorods at $400 \mathrm{~nm}$.
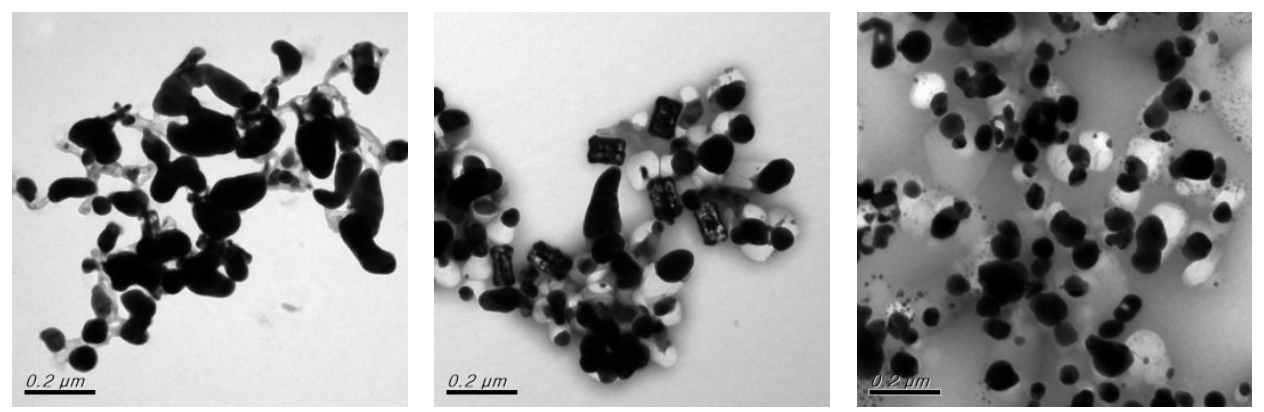

Figure S2. TEM images of the product in an attempt to synthesize Au-Ag nanozappers with excessive $\mathrm{HAuCl}_{4}$. The added $\mathrm{HAuCl}_{4}$ equivalent ratio was $120 \%$ to Ag atoms. Scale bars: $200 \mathrm{~nm}$. 

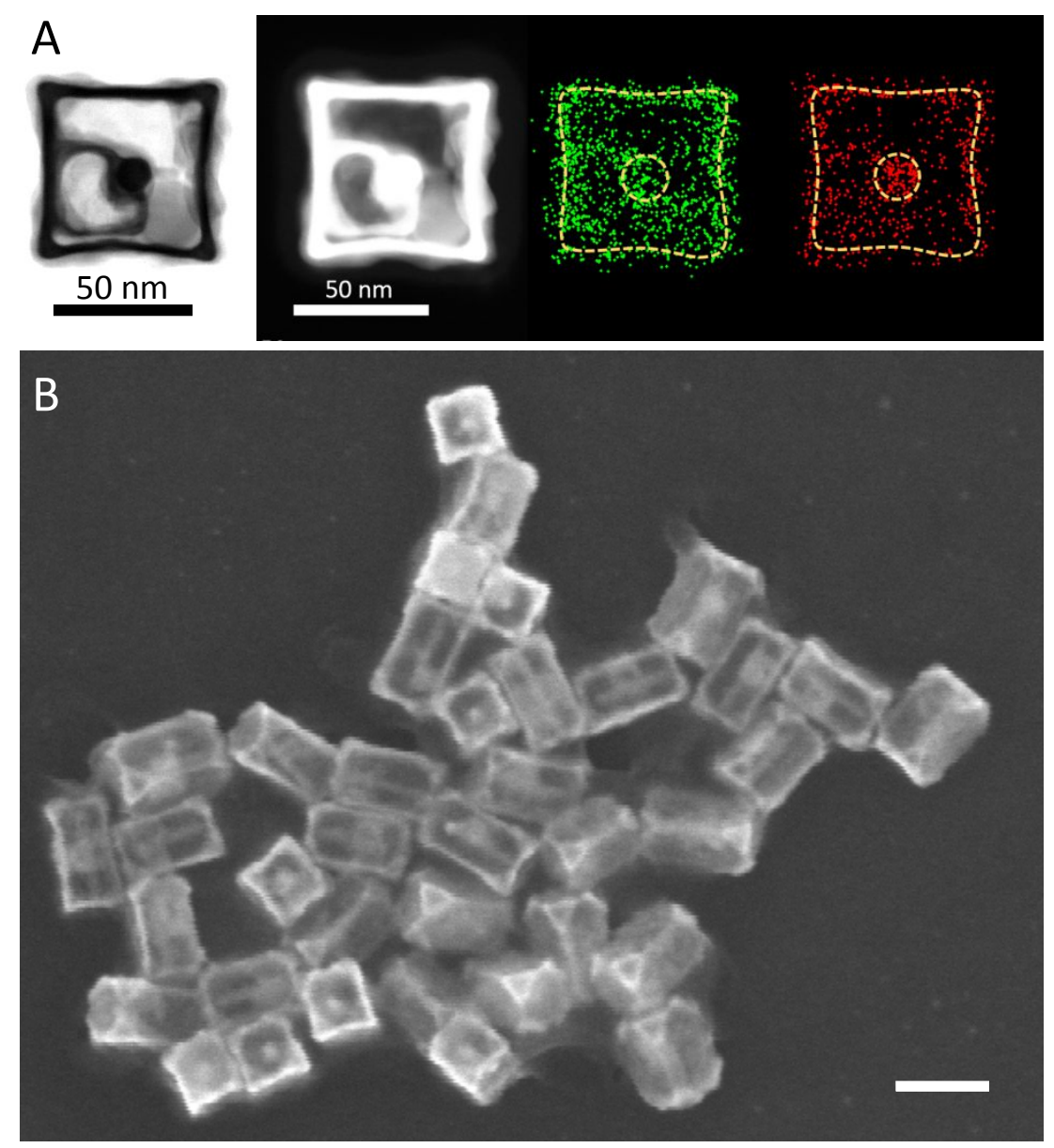

Figure S3. (A) Bright and dark field HAADF-STEM images and the corresponding EDS mappings of the top view of an Au-Ag nanozappers. The yellow dash lines outline the structures observed in STEM images. Scale bars: $50 \mathrm{~nm}$. (B) SEM image of Au-Ag nanozappers which show the 3-dimenstional structures. Scale bar: $100 \mathrm{~nm}$. 

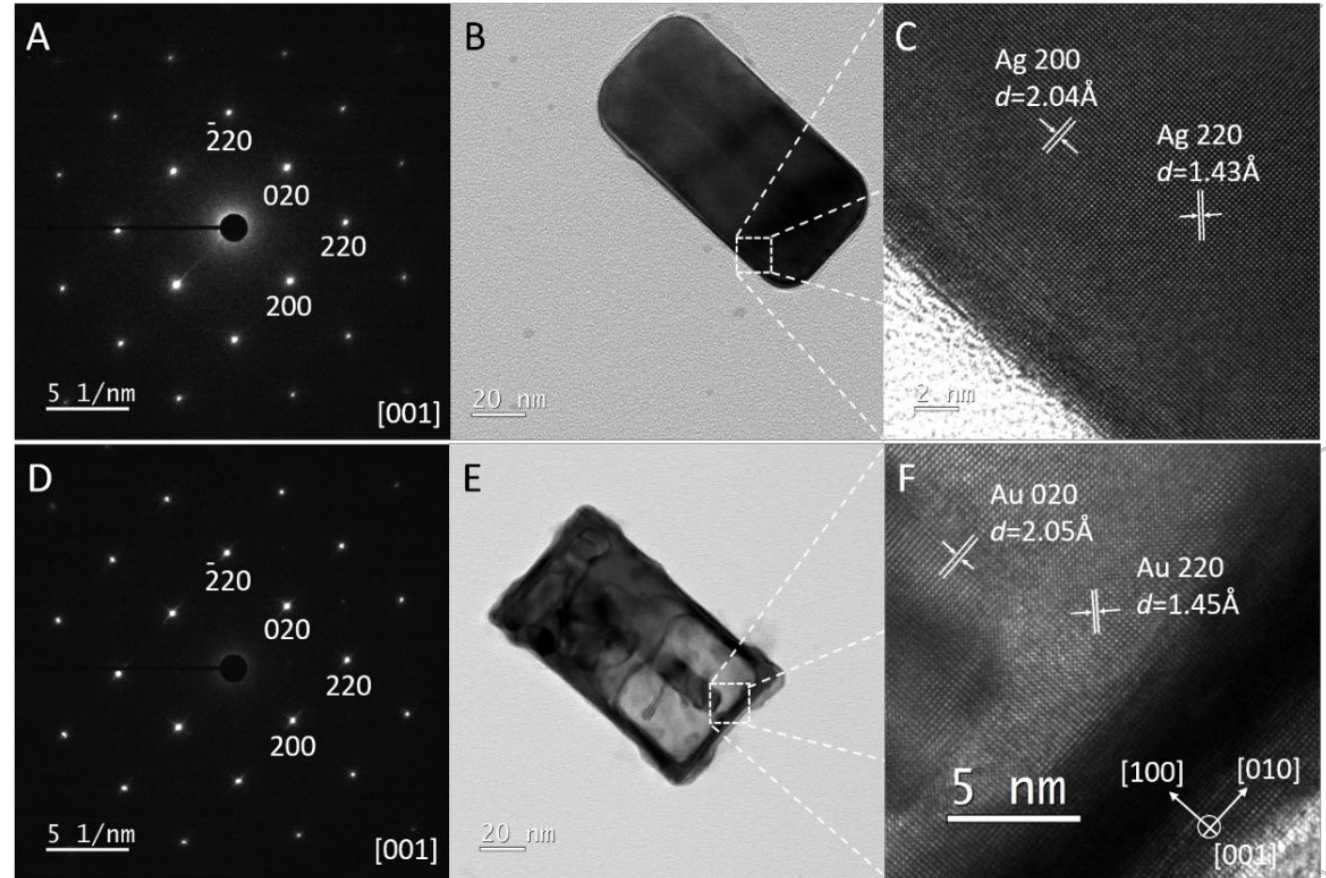

Figure S4. Crystallinities of Au@Ag nanocuboids (A, B, C) and Au-Ag nanozappers (D, E, F). (A, D) X-ray diffraction patterns taken from the zone highlighted in TEM images of (B) an Au@Ag nanocuboid and (E) an Au-Ag nanozapper. (C, F) HR-TEM images of (C) an Au@Ag nanocuboid and (F) Au-Ag nanozapper.

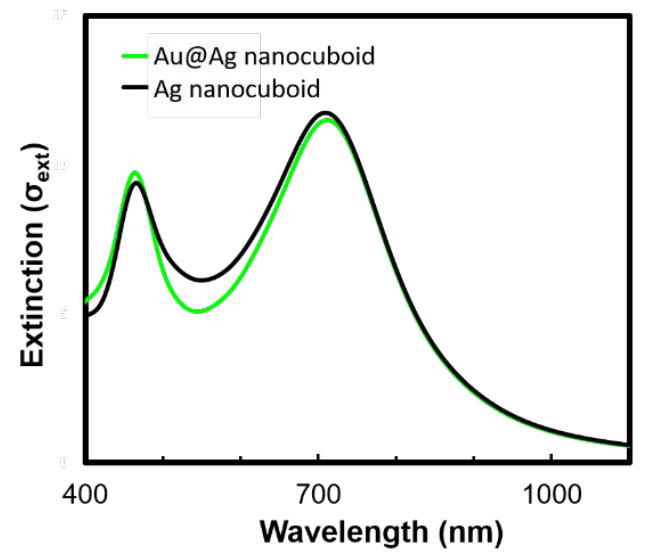

Figure S5. Simulated spectra of an Au@Ag nanocuboid (green) and Ag nanocuboid (black) with the same outer size. 


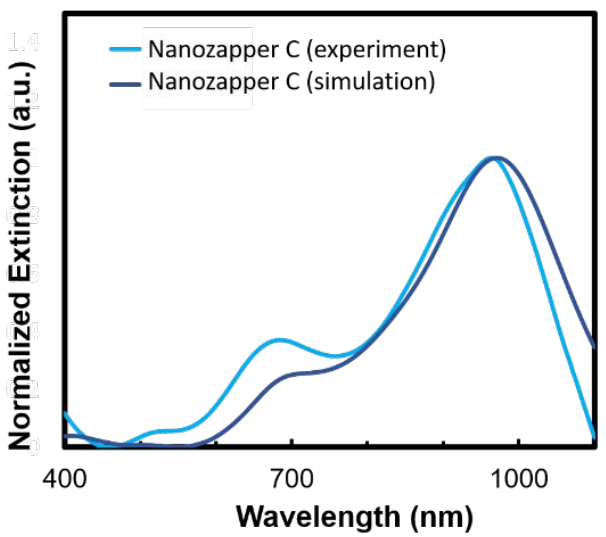

Figure S6. Experimental and simulated spectra of Nanozapper C.

Table S1. Size evolution of Au@Ag nanocuboid and Au-Ag nanozappers with $10 \%, 30 \%, 50 \%$ and 90\% equivalent ratio of added $\mathrm{HAuCl}_{4}$ to $\mathrm{Ag}$ in $\mathrm{Au@Ag} \mathrm{nanocuboid} \mathrm{solutions.}$

\begin{tabular}{llllll}
\hline $\begin{array}{l}\text { Added equivalent } \\
\text { ratio of } \mathrm{HAuCl}_{4}\end{array}$ & $\mathbf{0 \%}$ & $\mathbf{1 0 \%}$ & $\mathbf{3 0 \%}$ & $\mathbf{5 0 \%}$ & $\mathbf{9 0 \%}$ \\
\hline Length (nm) & $125.1 \pm 6.1$ & $126.3 \pm 6.5$ & $134.5 \pm 8.1$ & $138.4 \pm 7.2$ & $143.2 \pm 5.5$ \\
Width (nm) & $61.4 \pm 2.7$ & $61.0 \pm 4.4$ & $65.1 \pm 5.5$ & $70.2 \pm 4.5$ & $75.3 \pm 4.0$ \\
Aspect ratio & $2.04 \pm 0.62$ & $2.07 \pm 0.73$ & $2.07 \pm 0.79$ & $1.97 \pm 0.67$ & $1.90 \pm 0.52$ \\
\hline
\end{tabular}

\section{References}

1. Hendel, T.; Wuithschick, M.; Kettemann, F.; Birnbaum, A.; Rademann, K.; Polte, J. In Situ Determination of Colloidal Gold Concentrations with UV-Vis Spectroscopy: Limitations and Perspectives. Anan. Chem. 2014, 86, 1111511124.

2. Scarabelli, L.; Sánchez-Iglesias, A.; Pérez-Juste, J.; Liz-Marzán, L. M. A “Tips and Tricks" Practical Guide to the Synthesis of Gold Nanorods. J. Phys. Chem. Lett. 2015, 6, 4270-4279. 